\title{
Some Results on Rosa-type Labelings of Graphs
}

\author{
R. Rajarajachozhan*
}

\begin{abstract}
Labelings that are used in graph decompositions are called Rosa-type labelings. The $\gamma$-labeling of an almost-bipartite graph is a natural generalization of an $\alpha$-labeling of a bipartite graph. It is known that if a bipartite graph $G$ with $m$ edges possesses an $\alpha$-labeling or an almost-bipartite graph $G$ with $m$ edges possesses a $\gamma$-labeling, then the complete graph $K_{2 m x+1}$ admits a cyclic $G$-decomposition. A variation of an $\alpha$-labeling is introduced in this paper by allowing additional vertex labels and some conditions on edge labels and show that whenever a bipartite graph $G$ admits such a labeling, then there exists a supergraph $H$ of $G$ such that $H$ is almost-bipartite and $H$ has a $\gamma$-labeling.
\end{abstract}

Keywords: Graph Labeling, Rosa-type labeling, $\alpha$-labeling, $\gamma$-labeling, $\rho$ labeling

Mathematics Subject Classification (2010): 05C78

\section{Introduction}

Let $G$ be a simple connected bipartite graph with bipartition $(A, B)$ and $m$ edges. Let $V(G)$ and $E(G)$ denote the vertex set of $G$ and the edge set of $G$, respectively. Let $f: V(G) \rightarrow\{0,1,2, \ldots\}$ be a oneto-one function. Let $f(V(G))=\{f(v): v \in V(G)\}$. Define a function $\bar{f}: E(G) \rightarrow\{1,2,3, \ldots\}$ by $\bar{f}(e)=|f(u)-f(v)|$, where $e=u v \in E(G)$. We call $\mathrm{f}(\mathrm{v})$ and $\bar{f}(e)$ labels of the vertex $v$ and edge $e$ respectively. Let $\bar{E}(G)=\{\bar{f}(e): e \in E(G)\}$.

If $a$ and $b$ are integers with $a<b$, we denote $\{a, a+1, a+2, \ldots, b\}$ by $[a, b]$.

Rosa introduced a hierarchy of labelings including $\alpha$-labeling of a bipartite graph. [3] Blinco et al. introduced the notation of $\gamma$-labeling

*Research Scholar, Annamalai University, Tamil Nadu; r.rajarajachozhan@gmail.com 
of an almost-bipartite graph as a natural generalization of an $\alpha$-labeling of a bipartite graph.[1]

Joseph Gallian gives a comprehensive and updated dynamic survey on general graph labeling. [2]

Consider the following conditions.

$\ell_{1}: f(V(G)) \subseteq[0,2 m]$,

$\ell_{2}: f(V(G)) \subseteq[0, m]$,

$\ell_{3}: \bar{E}(G)=\left\{x_{1}, x_{2}, \ldots, x_{m}\right\}$, where for each $i \in[1, m]$ either $x_{i}=i$ or $x_{i}=2 m+1-i$,

$\ell_{4}: \bar{E}(G)=[1, m]$.

$\ell_{5}$ : there exists an integer $\lambda$ such that $f(a) \leq \lambda$ for all $a \in A$ and $f(b)>\lambda$ for all $b \in B$.

A labeling satisfying the conditions $\ell_{1}$ and $\ell_{3}$ is called a $\rho$-labeling. A labeling that satisfies $\ell_{2}, \ell_{4}$ and $\ell_{5}$ is called an $\alpha$-labeling.

A non-bipartite graph $G$ is said to be almost-bipartite if $G-e$ is bipartite for some $e \in E(G)$. If $G$ is almost-bipartite with $e=\hat{b} c$, then $G$ is tripartite and $V(G)$ can be partitioned into three sets $A, B$ and $\{c\}$ such that $\hat{b} \in B$ and $e$ is the only edge joining an element of $B$ to $c$.

Let $G$ be an almost-bipartite graph with $m$ edges with vertex tripartition $A, B,\{c\}$ as above. A labeling $h$ of the vertices of $G$ is called a $\gamma$-labeling of $G$ if the following conditions hold.

$g_{1}$ : The function $h$ is a $\rho$-labeling of $G$.

$g_{2}$ : If $a v$ is an edge of $G$ with $a \in A$, then $h(a)<h(v)$.

$g_{3}: h(c)-h(\hat{b})=m$.

It is known that

(i) Let $G$ be a graph with $m$ edges. There exists a cyclic $G$-decomposition of $K_{2 m+1}$ if and only if $G$ has a $\rho$-labeling. [3]

(ii) Let $G$ be a bipartite graph with $m$ edges that has an $\alpha$-labeling. Then, for all positive integers $x, G$ divides $K_{2 m x+1}$. [3]

(iii) Let $G$ be an almost-bipartite graph with $m$ edges having a $\gamma$-labeling. Then, for all positive integers $x, G$ divides $K_{2 m x+1}$.[1]

\section{Results}

If $\bar{f}$ is one-to-one and there exists an integer $\lambda$ such that $f(a) \leq \lambda$ for all $a \in A$ and $f(b)>\lambda$ for all $b \in B$, then we say that $f$ is an $\alpha(\bar{E}(G)$ )-labeling of $G$. When $f(V(G))=[0, m]$ an $\bar{E}(G)=[1, m]$, and $\alpha(\bar{E}(G)$ )-labeling of $G$ is the usual $\alpha$-labeling of $G$.

We now prove some results related to $\alpha$-labeling and $\lambda$-labeling.

Theorem 2.1. Let $f$ be an $\alpha(\bar{E}(G))$-labeling of $G$ with

$$
f(V(G)) \subseteq[0, m+1]
$$


and

$$
\bar{E}(G)=[1, m+1] \backslash\{d\},
$$

where $d \in[2, m]$. Suppose

$$
d=m+2-\lambda .
$$

Then there exist vertices $a \in A$ and $b \in B$, such that the almost-bipartite graph $G^{*}$ obtained from $G$ by defining

$$
V\left(G^{*}\right)=V(G) \cup\{v\}
$$

and

$$
E\left(G^{*}\right)=E(G) \cup\{a v, v b\}
$$

has a $\gamma$-labeling.

Proof. As $d \neq 1$ and $d \neq m+1$, there exist edges $e^{\prime}=x^{\prime} y^{\prime}$ with $x^{\prime} \in A$, $y^{\prime} \in B, f\left(x^{\prime}\right)=\lambda, f\left(y^{\prime}\right)=\lambda+1, \bar{f}\left(e^{\prime}\right)=1$ and $e^{\prime \prime}=x^{\prime \prime} y^{\prime \prime}$ with $x^{\prime \prime} \in A$, $y^{\prime \prime} \in B, f\left(x^{\prime \prime}\right)=0, f\left(y^{\prime \prime}\right)=m+1$ and $\bar{f}\left(e^{\prime \prime}\right)=m+1$. Take $a=x^{\prime \prime}$ and $b=y^{\prime}$. Then, $f(a)=0$ and $f(b)=\lambda+1$.

Define

$$
g: V\left(G^{*}\right) \rightarrow[0,2 m+4]
$$

by and

$$
g(w)=f(w) \text { for every } w \in V(G)
$$

$$
g(v)=\lambda+m+3
$$

Then

$$
\bar{g}(a v)=(2 m+5)-(\lambda+m+3)=m+2-\lambda=d
$$

and

$$
\bar{g}(v b)=(\lambda+m+3)-(\lambda+1)=m+2 .
$$

Hence,

$$
\bar{E}\left(G^{*}\right)=\bar{E}(G) \cup\{d, m+2\}=[1, m+2] .
$$

Clearly, $g$ is one-to-one and hence $g$ is a $\gamma$-labeling of $G^{*}$.

Theorem 2.2. Let $f$ be an $\alpha(\bar{E}(G))$-labeling of $G$ with

$$
f(V(G)) \subseteq[0, m+1]
$$

and

$$
\bar{E}(G)=[1, m+1] \backslash\{d\} .
$$

Suppose

$$
d=m+2-\lambda
$$


and there exists an $i$ in $[0, \lambda]$ such that both $i$ and $\lambda+1+i$ are in $f(V(G))$. Then there exist vertices $a \in A$ and $b \in B$, such that the almost-bipartite graph $G^{*}$ obtained from $G$ by defining

$$
V\left(G^{*}\right)=V(G) \cup\{v\}
$$

and

$$
E\left(G^{*}\right)=E(G) \cup\{a v, v b\}
$$

has a $\gamma$-labeling.

Proof. Take $a$ and $b$ so that $f(a)=i$ and $f(b)=\lambda+1+i$.

Define

$$
g: V\left(G^{*}\right) \rightarrow[0,2 m+4]
$$

by and

$$
g(w)=f(w) \text { for every } w \in V(G)
$$

$$
g(v)=\lambda+m+3+i
$$

Then

$$
\bar{g}(a v)=(2 m+5+i)-(\lambda+m+3+i)=m+2-\lambda=d
$$

and

$$
\bar{g}(v b)=(\lambda+m+3+i)-(\lambda+1+i)=m+2 .
$$

Hence,

$$
\bar{E}\left(G^{*}\right)=\bar{E}(G) \cup\{d, m+2\}=[1, m+2] .
$$

Clearly, $g$ is one-to-one and hence $g$ is a $\gamma$-labeling of $G^{*}$.

Remark 2.3. Theorem 2.2 is a generalization of Theorem 2.1. To see this, take $i=0$ in Theorem 2.2.

Theorem 2.4. Let $f$ be an $\alpha(\bar{E}(G))$-labeling of $G$ with

$$
f(V(G)) \subseteq[0, m+3] \backslash\{m+2\}
$$

and

$$
\bar{E}(G)=[1, m+2] \backslash\{d, m+4-d\}
$$

for some $d \in[2, m+2]$. Then, the almost-bipartite graph $G+K_{3}$, the disjoint union of $G$ and $K_{3}$, has a $\gamma$-labeling.

Proof. Let

$$
V\left(G+K_{3}\right)=V(G) \cup\{u, v, w\}
$$

and

$$
E\left(G+K_{3}\right)=E(G) \cup\{u v, v w, w u\} .
$$

Define

$$
g: V\left(G+K_{3}\right) \rightarrow[0,2 m+6]
$$


by

$$
\begin{aligned}
& g(x)=f(x)+d+1 \text { for every } x \in V(G) \\
& g(u)=0 \\
& g(v)=d
\end{aligned}
$$

and

Then,

$$
g(w)=m+d+3 .
$$

$$
\begin{gathered}
\bar{g}(u v)=d, \\
\bar{g}(v w)=(m+d+3)-d=m+3,
\end{gathered}
$$

and

$$
\bar{g}(w u)=(2 m+7)-(m+d+3)=m+4-d .
$$

Hence,

$$
\bar{E}\left(G+K_{3}\right)=\bar{E}(G) \cup\{d, m+4-d, m+3\}=[1, m+3] .
$$

Clearly, $g$ is one-to-one and hence $g$ is a $\gamma$-labeling of $G+K_{3}$.

Theorem 2.5. Let $f$ be an $\alpha(\bar{E}(G)$ )-labeling of $G$ with

$$
f(V(G)) \subseteq[0, m+3] \backslash\{m+1\}
$$

and

$$
\bar{E}(G)=[1, m+2] \backslash\{d, m+4-d\}
$$

for some $d \in[2, m+1]$. Then, the almost-bipartite graph $G+K_{3}$, the disjoint union of $G$ and $K_{3}$, has a $\gamma$-labeling.

Proof. In the proof of previous theorem, take $g(x)=f(x)+d+2$ for every $x \in V(G)$.

For integers $r \geq s \geq 2$, let $T_{r, s}$ be the double star with

$$
V\left(T_{r, s}\right)=\left\{x, y, x_{1}, x_{2}, \ldots, x_{r}, y_{1}, y_{2}, \ldots, y_{s}\right\}
$$

and

$$
E\left(T_{r, s}\right)=\left\{x y, x x_{1}, x x_{2}, \ldots, x x_{r}, y y_{1}, y y_{2}, \ldots, y y_{s}\right\} .
$$

Then, $m=r+s+1$.

We now see two illustrations associated with the above theorems.

Illustration 1 Define

$$
f: V\left(T_{r, s}\right) \rightarrow[0, m+1]
$$

by

$$
\begin{aligned}
& f(x)=0, \\
& f\left(y_{i}\right)=i \text { for every } i \in[1, s] \\
& f(y)=s+1
\end{aligned}
$$


and

$$
\left\{f\left(x_{1}\right), f\left(x_{2}\right), \ldots, f\left(x_{r}\right)\right\}=[s+2, r+s+2] \backslash\{r+3\} .
$$

So $\lambda=s$ and $d=m+2-\lambda=(r+s+1)+2-s=r+3$.

Then $f$ satisfies the hypothesis of Theorem 2.1. Hence, by the proof of Theorem 2.1, the almost-bipartite graph $T_{r, s}^{*}$ obtained from $T_{r, s}$ by defining

$$
V\left(T_{r, s}^{*}\right)=V\left(T_{r, s}\right) \cup\{v\}
$$

and

$$
E\left(T_{r, s}^{*}\right)=E\left(T_{r, s}\right) \cup\{x v, v y\}
$$

has a $\gamma$-labeling. Consequently, for all positive integers $x, T_{r, s}^{*}$ divides the complete graph $K_{2(r+s+3) x+1}$.

We apply Theorem 2.2 with $i=1$.

As $r \geq s, s+2 \neq r+3$, and hence $f\left(x_{1}\right)=s+2$. So, $f$ satisfies the hypothesis of Theorem 2.2. By the proof of Theorem 2.2, the almost-bipartite graph $T_{r, s}^{* *}$ obtained from $T_{r, s}$ by defining

$$
V\left(T_{r, s}^{* *}\right)=V\left(T_{r, s}\right) \cup\{v\}
$$

and

$$
E\left(T_{r, s}^{* *}\right)=E\left(T_{r, s}\right) \cup\left\{y_{1} v, v x_{1}\right\}
$$

has a $\gamma$-labeling. Consequently, for all positive integers $x, T_{r, s}^{* *}$ divides the complete graph $K_{2(r+s+3) x+1}$.

Illustration 2 Define

$$
f: V\left(T_{r, s}\right) \rightarrow[0, m+3]
$$

by

$$
\begin{aligned}
& f(x)=0, \\
& \left\{f\left(y_{1}\right), f\left(y_{2}\right), \ldots, f\left(y_{s}\right)\right\},=[1, s+1] \backslash\{s-1\}, \\
& f(y)=s+2,
\end{aligned}
$$
and

$$
\left\{f\left(x_{1}\right), f\left(x_{2}\right), \ldots, f\left(x_{r}\right)\right\},=[s+3, r+s+4] \backslash\{r+s+2\} .
$$

Then $f$ satisfies the hypothesis of Theorem 2.3 with $d=3$. Hence, by Theorem 2.3, the almost-bipartite graph $T_{r, s}+K_{3}$ has a $\gamma$-labeling. Consequently, for all positive integers $x, T_{r, s}+K_{3}$ divides the complete graph $K_{2(r+s+4) x+1}$.

The results obtained may be helpful to enlarge the known class of $\gamma$-labelable graphs. 
Rajarajachozhan

Rosa-type Labelings

\section{Acknowledgement}

The author wishes to thank R. Sampathkumar for his useful comments.

\section{References}

[1] A. Blinco, S. I. El-Zanati and C. V. Eynden, "On the cyclic decomposition of complete graphs into almost-bipartite graphs," Discrete Math., vol. 284, pp. 7181, 2004.

[2] J. A. Gallian, "A dynamic survey of graph labeling," DS\#6, Electron. J. Combin., pp. 1-408, Dec. 2016.

[3] A. Rosa, "On certain valuations of the vertices of a graph," in Theory of Graphs, Int. Sympos., Rome, 1966, ed. P. Rosenstiehl, Dunod, Paris; Gordon and Breach, New York, 1967, pp. 349-355. 